\title{
THE -REPRESENTATIONS OF SYMMETRIC HOMOGENEOUS ALGEBRAS
}

\author{
J. A. WARD \\ (Received 9 February 1984) \\ Communicated by W. Moran
}

\begin{abstract}
In 1947 I. E. Segal proved that to each non-degenerate $\sim$-representation $R$ of $L^{1}\left(=L^{1}(G)\right.$ for a compact group $G$ ) with representation space $\mathscr{H}$, there corresponds a continuous unitary representation $W$ of $G$, also with representation space $\mathscr{H}$, which satisfies

$$
\langle R(f) h, k\rangle=\int_{G}\langle W(x) h, k\rangle f(x) d x
$$

for each $f \in L^{1}$ and $h, k \in \mathscr{H}$. This was extended to $L^{p}, 1 \leqslant p<\infty$, in 1970 by E. Hewitt and $\mathrm{K}$. A. Ross. We now generalize this result to any symmetric homogeneous convolution Banach algebra of pseudomeasures on $G$. Further we prove that the correspondence preserves irreducibility.
\end{abstract}

1980 Mathematics subject classification (Amer. Math. Soc.): 22 D 20; secondary 43 A 65.

Throughout $G$ will denote a compact group with dual object $\Sigma$. As usual $\sigma$ will denote an arbitrary element of $\Sigma$, and $U_{\sigma}$ a representation in $\sigma$ acting on the Hilbert space $\mathscr{H}_{\sigma}$. For each $p, 1 \leqslant p<\infty, L^{p}$ will denote the Banach space of $p$-integrable functions on $G$ with respect to Haar measure. It is well known that $L^{p}$ is a symmetric Banach algebra if multiplication is convolution and the involution $\sim$ is defined by $\tilde{f}(x)=\overline{f\left(x^{-1}\right)}$ for all $x \in G$ and $f \in L^{p}$.

For any Hilbert space $\mathscr{H}, \mathscr{B}(\mathscr{H})$ will denote the space of bounded linear operators on $\mathscr{H}$, and \|\| the usual operator norm. If $\mathscr{B}(\mathscr{H})$ is involuted with the adjoint map, then it also is a symmetric Banach algebra.

We define a $\sim$-representation $R$ of $L^{p}$ on $\mathscr{H}$ to be any representation which preserves involution; it is non-degenerate if for each non-zero $h \in \mathscr{H}$ there exists $f \in L^{p}$ such that $R_{f} h \neq 0$, where $R_{f}$ denotes $R(f)$.

(c) 1986 Australian Mathematical Society $0263-6115 / 86 \$ A 2.00+0.00$ 
Segal [4] proved that to each non-degenerate -representation $R$ of $L^{1}$ there corresponds a unique continuous unitary representation $W$ of $G$, both $R$ and $W$ acting on a Hilbert space $\mathscr{H}$, such that

$$
\left\langle R_{f} h, k\right\rangle=\int_{G}\left\langle W_{x} h, k\right\rangle f(x) d x
$$

for all $h, k \in \mathscr{H}$ and $f \in L^{1}$. Hewitt and Ross [2] extend this result in (38.21) to $L^{p}$ for $1 \leqslant p<\infty$.

We shall derive a corresponding result for all symmetric convolution Banach algebras of pseudomeasures on $G$. Our results will include those of Segal, Hewitt and Ross since each $L^{p}, 1 \leqslant p<\infty$, is such a Banach algebra.

We will denote by $A$ the space of continuous functions on $G$ which possess absolutely summable Fourier transforms, and by $P$ its continuous dual. The elements of $P$ are called pseudomeasures.

Ward [5], [6] detail the properties of $P$ and its elements. However, for convenience we provide a short summary. The Fourier transform $\hat{S}(\sigma)$ of an element $S$ of $P$ at the point $\sigma$ of $\Sigma$ is defined to be the operator $S_{\sigma}$ in $\mathscr{B}\left(\mathscr{H}_{\sigma}\right)$ which satisfies

$$
S(f)=\sum_{\sigma \in \Sigma} d_{\sigma} \operatorname{tr}\left(S_{\sigma} \hat{f}(\sigma)^{*}\right)
$$

for all $f \in A$. We note that $\sup _{\sigma \in \Sigma}\left\|S_{\sigma}\right\|$ is finite and that $\|S\|_{\mathbf{P}}=\sup _{\sigma \in \Sigma}\left\|S_{\mathrm{o}}\right\|$, so that $S$ can be identified with the sequence $\left(S_{\sigma}\right)_{\sigma \in \Sigma}$. For each $x \in G$, the left $x$-translate ${ }_{x} S$ of $S$ is defined by ${ }_{x} S(f)=S\left(x_{x^{-1}} f\right)$ for all $f \in A$. The involution $\sim$ and convolution product $*$ are defined on $\mathbf{P}$ by $(\tilde{S})_{\sigma}=S_{\sigma}^{*}$ and $(S * T)_{\sigma}=$ $S_{\sigma} T_{\sigma}$, respectively, for each $\sigma \in \Sigma$.

Throughout $B$ will denote a symmetric homogeneous convolution Banach algebra of pseudomeasures, and \|\|$_{B}$ its norm. The homogeneity means that $B$ is left translation invariant, that each left translation operator is continuous on $B$, and that the map $x \rightarrow_{x} b$ is continuous on $G$ for each $b \in B$. It is further assumed that the injection of $B$ into $P$ is continuous.

As already noted each $L^{p}, 1 \leqslant p<\infty$, is homogeneous. Further examples are $A, C$ (the space of continuous functions on $G$ ) and, for $1 \leqslant p<\infty, U^{p}$ (the space of $L^{1}$-functions with $p$-summable Fourier transforms). For a discussion of these and other examples see Section 5 of [5]. It is, in fact, shown in [5, (3.1)] that for any subset $F$ of $\Sigma$

$$
B_{F}=\{b \in B: \operatorname{supp}(\hat{b}) \subseteq F\}
$$

is a closed symmetric subalgebra of $B$, and so is also homogeneous. This provides us with a method of producing an abundance of non-trivial examples. 
It is an important consequence of 2.4 and 2.6 of [5] that there exists a subset $F$ of $\Sigma$ such that $T_{F}$ (the set of trigonmetric polynomials with Fourier transforms supported by $F$ ) is a dense subspace of $B$. Consequently, for each $\sigma \in F, B_{\{\sigma\}}$ is isomorphic to $\mathscr{B}\left(\mathscr{H}_{\sigma}\right)$.

We now let $R$ denote a non-degenerate $\sim$-representation of $B$ acting on some Hilbert space $\mathscr{H}$. Then, for each $\sigma \in F, R$ induces a $\sim$-representation $Q_{\mathrm{o}}$ of $\mathscr{B}\left(\mathscr{H}_{\sigma}\right)$ on $\mathscr{H}$ by

$$
Q_{\sigma}(T)=R\left(x \rightarrow d_{\sigma} \operatorname{tr}\left(T U_{\sigma}(x)^{*}\right)\right)
$$

for each $T \in \mathscr{B}\left(\mathscr{H}_{\sigma}\right)$.

Naimark [3] has completely determined the structure of $\sim$-representations of finite dimensional operator algebras $\mathscr{B}(\mathscr{K})$. It follows from [3, (22.2)] that, for each $\sigma \in F$, there exists a family of mutually orthogonal $Q_{\sigma}$-invariant closed subspaces $\mathscr{K}_{\sigma}^{0}, \mathscr{K}_{\sigma}^{a}, a \in \mathscr{A}$, of $\mathscr{H}$ satisfying

(i) $\mathscr{H}=\mathscr{K}_{0}^{0} \oplus \underset{a \in \mathscr{A}}{\bigoplus_{\sigma}} \mathscr{K}^{a}$,

(ii) $\operatorname{dim} \mathscr{K}_{\mathrm{a}}^{a}=\operatorname{dim} \mathscr{H}_{\mathrm{o}} \stackrel{\mathrm{df}}{=} d_{\sigma}$ for each $a \in \mathscr{A}$, and

(iii) $\mathscr{K}_{\sigma}^{0}=\bigcap\left\{\operatorname{ker} Q_{\sigma}(T): T \in \mathscr{B}\left(\mathscr{H}_{\sigma}\right)\right\}$, and a family, indexed by $\mathscr{A}$, of unitary operators $V_{\sigma}^{a}: \mathscr{H}_{\sigma} \rightarrow \mathscr{K}_{\sigma}^{a}$ such that, for each $T \in \mathscr{B}\left(\mathscr{H}_{\sigma}\right), Q_{\sigma}(T)$ can be identified with the direct sum

$$
\bigoplus_{a \in \mathscr{A}} V_{\mathrm{o}}^{a} T V_{\sigma}^{a^{*}}
$$

That is, for each $h \in \oplus_{a \in \mathscr{A}} \mathscr{K}_{\sigma}^{a}$, regarded as a subset of $\mathscr{H}$, we have

$$
Q_{\mathrm{o}}(T) h=\left(\bigoplus_{a \in \mathscr{\infty}} V_{\sigma}^{a} T V_{\sigma}^{a^{*}}\right) h
$$

It follows that if $P_{\sigma}^{a}$ denotes the orthogonal projection of $\mathscr{H}$ onto $\mathscr{K}_{\sigma}^{a}$, then the series $\sum_{a \in \mathscr{A}} V_{\sigma}^{a} T V_{\sigma}^{a^{*}} P_{\sigma}^{a}$ is convergent in $\mathscr{B}(\mathscr{H})$ in the strong operator topology.

We shall use this decomposition of the $Q_{\sigma}$ to obtain a decomposition of $R$ which will suggest an 'obvious' choice for $W$. First, however, we require a preliminary result concerning the orthogonality of the set of projections $\left\{P_{\sigma}^{a}: a \in \mathscr{A}, \sigma \in F\right\}$.

LEMMA 1. $\left\{P_{\sigma}^{a}: a \in A, \sigma \in F\right\}$ is orthogonal.

Proof. Since $\mathscr{H}$ is the direct sum of $\mathscr{K}_{\sigma}^{0}$ and the $\mathscr{K}_{\sigma}^{a}$ 's it is clear that for each $\sigma \in F,\left\{P_{\mathrm{o}}^{a}: a \in \mathscr{A}\right\}$ is orthogonal. So it is sufficient to show that, provided $\eta \neq \sigma$, we have $\oplus_{a \in \mathscr{A}} \mathscr{K}_{\sigma}^{a} \subseteq \mathscr{K}_{\eta}^{0}$. 
Assume now that $\eta \neq \sigma$. Observe that for each $b_{1} \in B_{\{\eta\}}$ and $b_{2} \in B_{\{\sigma\}}$, we have $R\left(b_{1} * b_{2}\right)=R(0)=0$, and so $Q_{\eta}\left(T_{1}\right) Q_{\sigma}\left(T_{2}\right)=0$ for each $T_{1} \in \mathscr{B}\left(\mathscr{H}_{\eta}\right)$ and $T_{2} \in \mathscr{B}\left(\mathscr{H}_{\sigma}\right)$. We observe that if $T_{2}=I_{\sigma}=$ the identity map on $\mathscr{H}_{\sigma}$, then $Q_{\sigma}\left(I_{\sigma}\right)$ $=\Sigma_{a \in \mathscr{A}} P_{\sigma}^{a}$, and so $Q_{\eta}\left(T_{1}\right) h=0$ for all $T_{1} \in \mathscr{B}\left(\mathscr{H}_{\eta}\right)$ and $h \in \oplus_{a \in \mathscr{A}} \mathscr{K}_{\sigma}^{a}$. Hence $\oplus_{a \in \mathscr{A}} \mathscr{K}_{\sigma}^{a} \subseteq \mathscr{X}_{\eta}^{0}$.

We observe that, for each $\sigma \in F$ and $a \in \mathscr{A}$, the operator $V_{a}^{a} T V_{\sigma}^{a^{*}}$ is an operator in $\mathscr{B}\left(\mathscr{K}_{\sigma}^{a}\right)$ with $\left\|V_{\sigma}^{a} T V_{\sigma}^{a^{*}}\right\| \leqslant\|T\|$. It follows from Lemma 1 that if $\left(T_{\sigma}\right)_{\sigma \in F}$ denotes a set of operators, $T_{\sigma} \in \mathscr{B}\left(\mathscr{H}_{\sigma}\right)$, with $\sup _{\sigma \in F}\left\|T_{\sigma}\right\|<\infty$, then $\oplus_{\sigma \in F} \oplus_{a \in \mathscr{A}} V_{a}^{a} T_{\sigma} V_{\sigma}^{a^{*}}$ is a bounded operator in $\mathscr{B}(\mathscr{H})$. We can now prove that the representation $R$ can be reconstructed using the decomposition of the $Q_{\sigma}$ 's.

THEOREM 1. For each $b \in B$, we have

$$
R(b)=\bigoplus_{\sigma \in F} \bigoplus_{a \in \mathscr{A}} V_{\sigma}^{a} \hat{b}(\sigma) V_{\sigma}^{a^{*}}
$$

PROof. If $b$ is a trigonometric polynomial, then the support of $\hat{b}$ is a finite subset $F_{0}$ of $F$, and so

$$
\begin{aligned}
R(b) & =\sum_{\sigma \in F_{0}} R\left(x \rightarrow d_{\sigma} \operatorname{tr}\left(\hat{b}(\sigma) U_{\sigma}(x)^{*}\right)\right) \\
& =\sum_{\sigma \in F_{0}} Q_{\sigma}(\hat{b}(\sigma)) \\
& =\bigoplus_{\sigma \in F_{0}} \bigoplus_{a \in \mathscr{A}} V_{\sigma}^{a} \hat{b}(\sigma) V_{\sigma}^{a^{*}} \\
& =\bigoplus_{\sigma \in F} \bigoplus_{a \in \mathscr{A}} V_{\sigma}^{a} \hat{b}(\sigma) V_{\sigma}^{a^{*}} .
\end{aligned}
$$

Now let $\left(k_{\lambda}\right)_{\lambda \in \Lambda}$ denote an approximate identity in $L^{1}$. It follows from [5, (2.5)] and from the continuity of $R$ that, for each $b \in B$,

$$
\lim _{\lambda \in \Lambda}\left\|R(b)-R\left(k_{\Lambda^{*}} b\right)\right\|=0 .
$$

In particular, if each $k_{\lambda}$ is a trigonometric polynomial then $k_{\lambda} * b \in T_{F}$ and so

$$
R\left(k_{\lambda} * b\right)=\bigoplus_{\sigma \in F} \bigoplus_{a \in \mathscr{A}} V_{\sigma}^{a}\left(k_{\lambda} * b\right)^{\wedge}(\sigma) V_{\sigma}^{a^{*}} .
$$

We note that for each $b \in B$, we have

$$
\sup _{\sigma \in F}\|\hat{b}(\sigma)\|=\|b\|_{P} \leqslant C\|b\|_{B}
$$

and so, as our discussion following Lemma 1 indicates, $\oplus_{\sigma \in F} \oplus_{a \in \mathscr{A}} V_{\sigma}^{a} \hat{b}(\sigma) V_{\sigma}^{a^{*}}$ is an operator in $\mathscr{B}(\mathscr{H})$. Therefore, it follows from (3) that (2) holds, provided that

$$
\lim _{\lambda \in \Lambda}\left\|\bigoplus_{\sigma \in F} \bigoplus_{a \in \mathscr{A}} V_{\sigma}^{a}\left(\hat{b}(\sigma)-\left(k_{\lambda} * b\right)^{\wedge}(\sigma)\right) V_{\sigma}^{a^{*}}\right\|=0
$$


That this limit exists and is equal to 0 is a consequence of the inequality

$$
\begin{aligned}
\left\|\sum_{\sigma \in F} \sum_{a \in \mathscr{A}} V_{\sigma}^{a}\left[\hat{b}(\sigma)-\left(k_{\lambda} * b\right)^{\wedge}(\sigma)\right] V_{\sigma}^{a^{*}} P_{\sigma}^{a} h\right\|_{\mathscr{H}}^{2} \\
\quad=\sum_{\sigma \in F} \sum_{a \in \mathscr{A}}\left\|V_{\sigma}^{a}\left[\hat{b}(\sigma)-\left(k_{\lambda} * b\right)^{\wedge}(\sigma)\right] V_{\sigma}^{a^{*}} P_{\sigma}^{a} h\right\|_{\mathscr{H}}^{2} \cdot \\
\quad \leqslant \sup _{\sigma \in \Sigma, a \in \mathscr{A}}\left\|V_{\sigma}^{a}\left[\hat{b}(\sigma)-\left(k_{\lambda} * b\right)^{\wedge}(\sigma)\right] V_{\sigma}^{a^{*}}\right\| \sum_{\sigma \in \Sigma} \sum_{a \in \mathscr{A}}\left\|P_{\sigma}^{a} h\right\|_{\mathscr{H}}^{2} \\
\leqslant\left\|b-k_{\lambda} * b\right\|_{B}^{2}\|h\|_{\mathscr{H}}^{2}
\end{aligned}
$$

for all $b \in B$.

Theorem 1 together with Equation (1) establishes that for each $b \in B$,

$$
R(b)=\sum_{\sigma \in F} \sum_{a \in \mathscr{A}} V_{a}^{a} \hat{b}(\sigma) V_{\sigma}^{a^{*}} P_{\sigma}^{a},
$$

the series being convergent in the strong operator topology on $\mathscr{B}(\mathscr{H})$.

If we recall our intention to generalize Segal's result, this suggests that the sums $\sum_{\sigma \in F} \sum_{a \in \mathscr{A}} V_{\sigma}^{a} U_{\sigma}(x) V_{\sigma}^{a *} P^{a}$ may be useful. Each is convergent in the strong operator topology on $\mathscr{B}(\mathscr{H})$ since, for each $x \in G$, we have

$$
\begin{aligned}
\left\|\sum_{\sigma \in F} \sum_{a \in \mathscr{A}} V_{\sigma}^{a} U_{\sigma}(x) V_{\sigma}^{a^{*}} P_{\sigma}^{a} h\right\|_{\mathscr{H}}^{2} & =\sum_{\sigma \in F} \sum_{a \in \mathscr{A}} \| V_{\sigma}^{a} U_{\sigma}(x) V_{\sigma}^{a^{*} P_{\sigma}^{a} h \|_{\mathscr{H}}^{2}} \\
& \leqslant \sum_{\sigma \in F} \sum_{a \in \mathscr{A}}\left\|P_{\sigma}^{a} h\right\|_{\mathscr{H}}^{2}=\|h\|_{\mathscr{H}}^{2}
\end{aligned}
$$

for each $h \in \mathscr{H}$.

For each $x \in G$ we define $W(x)$ by

$$
W(x)=\bigoplus_{\sigma \in F} \bigoplus_{a \in \mathscr{Q}} V_{\sigma}^{a} U_{0}(x) V_{\sigma}^{a^{*}},
$$

which is in $\mathscr{B}(\mathscr{H})$ because $\sup _{\sigma \in F, a \in \mathscr{A}}\left\|V_{\sigma}^{a} U_{\sigma}(x) V_{\sigma}^{a^{*}}\right\| \leqslant 1$. Then, for each $h \in \mathscr{H}$, we have

$$
W(x) h=\sum_{\sigma \in F} \sum_{a \in \mathscr{A}} V_{\sigma}^{a} U_{\sigma}(x) V_{\sigma}^{a *} P_{\sigma}^{a} h,
$$

where, by (4), the series must be uniformly convergent in $\mathscr{H}$ over $G$.

Lemma 2. The map $W: G \rightarrow \mathscr{B}(\mathscr{H})$, defined by $x \mapsto W(x)$, is a continuous unitary representation of $G$ on $\mathscr{H}$.

ProOf. First observe that since $R$ is non-degenerate we have

$$
\bigoplus_{\sigma \in F} \bigoplus_{a \in \mathscr{A}} \mathscr{K}_{\sigma}^{a}=\mathscr{H}
$$


and so $\sum_{o \in F} \Sigma_{a \in \mathscr{A}} P_{a}^{a}=I$, the identity map on $\mathscr{H}$. Consequently each operator $W(x)$ is unitary. Furthermore, by Lemma $1, W$ must be multiplicative, and so it remains only to prove that $W$ is continuous. In fact, as $W$ is unitary it is sufficient to prove that it is weakly continuous.

Now, for each $\sigma \in F, a \in \mathscr{A}, h \in \mathscr{H}$ and $x, y \in G$, we have

$$
\left\|V_{\sigma}^{a}\left[U_{\sigma}(x)-U_{\sigma}(y)\right] V_{\sigma}^{a^{*}} P_{\sigma}^{a} h\right\|_{\mathscr{H}} \leqslant\left\|U_{\sigma}(x)-U_{\mathrm{o}}(y)\right\|\|h\|_{\mathscr{H}}
$$

so that the functions $x \mapsto V_{\sigma}^{a} U_{\sigma}(x) V_{\sigma}^{a^{*}} P_{\sigma}^{a} h$ are continuous from $G$ to $\mathscr{H}$. Since the series $\sum_{\sigma \in F} \sum_{a \in \mathscr{A}} V_{\sigma}^{a} U_{\sigma}(x) V_{\sigma}^{a^{*}} P_{\sigma}^{a} h$ must be uniformly convergent in $\mathscr{H}$ over $G$, this guarantees weak continuity.

The theory of vector-valued integrals, as presented in [1, Sections 8.14 and 8.15 ], can be used to demonstrate that $R$ can be recovered from $W$. To this end, we again let $\left(k_{\lambda}\right)_{\lambda \in \Lambda}$ denote an approximate identity, consisting of trigonometric polynomials of $L^{1}$. We then prove:

TheOREM 2. For each $b \in B, R(b)=\lim _{\lambda \in \Lambda} \int_{G} k_{\lambda} * b(x) W(x) d x$ in $\mathscr{B}(\mathscr{H})$.

Proof. We first take $b \in T_{F}$ and use some familiar properties of vector-valued integrals to prove that $\int_{G} b(x) W(x) d x$ is an element of $\mathscr{B}(\mathscr{H})$. By $[1,8.15 .2$ and 8.14.4], it is sufficient to prove that the function $g: G \rightarrow \mathscr{B}(\mathscr{H}), x \rightarrow b(x) W(x)$, is measureable and that the real integral $\int_{G}\|g(x)\| d x$ is finite. However, observe that the first of these conditions follows from Lemma 2, and the second from Lemma 2 and the compactness of $G$.

We now prove that for such $b$,

$$
R(b)=\int_{G} b(x) W(x) d x
$$

We know that, for each $h \in \mathscr{H}$,

$$
\begin{aligned}
R(b) h & =\sum_{\sigma \in F} Q_{\sigma}(\hat{b}(\sigma)) h \\
& =\sum_{\sigma \in F} \sum_{a \in \mathscr{A}} V_{\sigma}^{a}\left[\int_{G} b(x) U_{\sigma}(x) d x\right] V_{\sigma}^{a^{*}} P_{\sigma}^{a} h,
\end{aligned}
$$

and that each of the functions $x \rightarrow V_{\sigma}^{a} b(x) U_{\sigma}(x) V_{\mathrm{o}}^{a^{*}} P_{\mathrm{\sigma}}^{a} h$ is continuous from $G$ to $\mathscr{H}$. Since the series is uniformly convergent in $\mathscr{H}$ over $G$, we can interchange the order of summation and integration in (6), giving (5).

To extend the result to the whole of $B$, let $b$ denote an arbitrary element of $B$ and $\left(k_{\lambda}\right)_{\lambda \in \Lambda}$ an approximate identity, consisting of trigonometric polynomials, of $L^{1}$. Then for each $\lambda \in \Lambda, k_{\lambda} * b$ is a trigonometric polynomial, and so it 
follows from (3) and (5) that

$$
\lim _{\lambda \in \Lambda}\left\|R(b)-\int_{G} k_{\lambda} * b(x) W(x) d x\right\|=0 .
$$

In particular we obtain the obvious extension of Segal's result.

COROllary. For each $b \in B$ and $h, k \in \mathscr{H}$,

$$
\langle R(b) h, k\rangle=b[x \rightarrow\langle W(x) h, k\rangle],
$$

where $b$ acts as a continuous linear functional on $A$. Moreover, if $b \in L^{1}$ then

$$
\langle R(b) h, k\rangle=\int_{G}\langle W(x) h, k\rangle b(x) d x .
$$

We also obtain the result of Hewitt and Ross as a corollary.

Corollary. If $B=L^{p}$ for some $p, 1 \leqslant p<\infty$, then for all $b \in B$,

$$
R(b)=\int_{G} b(x) W(x) d x
$$

in $\mathscr{B}(\mathscr{H})$.

PROOF. It follows from $[1,8.14 .6]$ that for each $b \in B$ and $\lambda \in \Lambda$,

$$
\left\|\int_{G}\left(k_{\lambda} * b-b\right)(x) W(x) d x\right\| \leqslant\left\|k_{\lambda} * b-b\right\|_{L^{p}} \sup _{x \in G}\|W(x)\| .
$$

Therefore, since

$$
\begin{aligned}
\left\|R(b)-\int_{G} b(x) W(x) d x\right\| \leqslant & \left\|R(b)-\int_{G} k_{\lambda} * b(x) W(x) d x\right\| \\
& +\left\|\int_{G}\left(k_{\lambda} * b-b\right)(x) W(x) d x\right\|,
\end{aligned}
$$

Theorem 2 ensures (7).

In the case of either group or of algebra representations, of fundamental importance are the so-called irreducible representations. In either case, a representation is called irreducible if the only subspaces of $\mathscr{H}$ which are invariant under the action of the representation are the trivial ones $\{0\}$ and $\mathscr{H}$. In other words, the only projections commuting with the action of the representation are 
the 0 and the identity operators. The continuous irreducible unitary representations of a group $G$ are identified, up to equivalence, with $\Sigma$. We now prove that the continuous irreducible unitary representations of $B$ are identified, up to equivalence, with $F$.

THEOREM 3. $R$ is irreducible if and only if $W$ is irreducible.

Proof. Assume that $R$ is reducible and let $\mathscr{K}$ denote a proper closed subspace of $\mathscr{H}$ which is invariant under the action of $R$. For each finite subset $E$ of $F$ define the complex-valued map $\omega_{E}$ on $G$ by $\omega_{E}(x)=\Sigma_{\sigma \in E} d_{\sigma} \operatorname{tr}\left(U_{\sigma}(x)^{*}\right)$. Then $\omega_{E} \in T_{F}$, and by Theorem 1 we have

$$
\lim _{E}\left\|W(x) h-R\left({ }_{x} \omega_{E}\right) h\right\|_{\mathscr{H}}=0
$$

for each $h \in \mathscr{H}$. But $R\left({ }_{x} \omega_{E}\right) \mathscr{K} \subseteq \mathscr{K}$ for each $x \in G$ and finite subset $E$ of $F$, and so $W(x) \mathscr{K} \subseteq \mathscr{K}$ for all $x \in G$. Hence $W$ is reducible. On the other hand, if $W$ is reducible, and $P$ denotes a non-trivial projection commuting with $W$, then $P W(x)=W(x) P$ for all $x \in G$. Hence, by Theorem $2 P R(b)=R(b) P$ for each $b \in B$, and so $R$ is also reducible.

So if $R$ is an irreducible unitary representation of $B$, then Theorem 3 ensures that $W=U_{\text {o }}$ for some $\sigma \in F$.

\section{Acknowledgements}

We wish to acknowledge Professor R. E. Edwards for several helpful discussions, and Dr. W. R. Bloom for his comments. In particular, we wish to thank the referee for his valuable comments and assistance in the final preparation of this paper.

\section{References}

[1] R. E. Edwards, Functional analysis, theory and applications (Holt, Rinehart and Winston, New York, 1965).

[2] E. Hewitt and K. A. Ross, Abstract harmonic analysis, Vols. 1 and 2 (Springer Verlag, Berlin, 1963 and 1970).

[3] M. A. Naimark, Normed rings (Noordhoff, Groningen, 1959). 
[4] I. E. Segal, 'The group algebra of a locally compact group', Trans. Amer. Math. Soc. 61 (1947), 69-105.

[5] J. A. Ward, Characterization of homogeneous spaces and their norms, Pacific J. Math. 114 (1984), 481-495.

[6] J. A. Ward, 'Closed ideals of homogeneous algebras', Monatsh. Math. 96 (1983), 317-324.

School of Mathematical and Physical Sciences

Murdoch University

Murdoch

Western Australia 6155

Australia 